\title{
Postsynaptic modulation of electrical EPSP size investigated using a compartmental model
}

\author{
Ovidiu D. Iancu, Patrick D. Roberts, Jianmei Zhang and Curtis C. Bell \\ Neurological Sciences Institute, Portland, Oregon
}

September 4, 2006

\begin{abstract}
The mormyrid electric fish displays extremely fine temporal resolution as measured in behavioral studies. The first stage of sensory processing, the sensory afferents, also display very precise temporal responses to electrical stimulation. The afferents are connected through gap junctions to the granular cells, which in addition receive a corollary discharge signal at the time of the electric pulse. The granular cells have a relatively long time constant, seemingly at odds with their presumed role as coincidence detectors. We use experimental data to build a compartmental model that investigates the mechanism by which the relative timing of the two inputs to the granular cells determines the effect of the afferent spike through the electrical synapse.
\end{abstract}

\section{Introduction}

Mormyrid fish emit short pulses of electricity from an electric organ in their tails. The electric field generated by these pulses interacts with objects in the immediate vicinity of the fish and activates electroreceptors on the skin. The electroreceptor afferents generate one or more spikes, with the timing of the first spike strongly correlated with the amplitude of the electric field. Behavioral studies suggest that the temporal sensitivity of the fish is extremely high (Hall et al., 1995). Objectinduced distortions of the field are sensed by the electroreceptors, thus providing the fish with sensory information about its environment.

Primary afferent axons from electroreceptors terminate centrally in the electrosensory lobe $(E L L)$, where they form electrical synapses on granular cells. The granular cells also receive excitatory descending input from the juxtalobar nucleus $(J L N)$, which conveys a precisely timed corollary discharge signal associated to the motor command that generates the electric pulse. Interaction between the variable afferent input and the fixed latency corollary discharge signal is thought to be the means by which afferent latency is decoded as a measure of stimulus intensity (Bell et al., 1992) .

In vitro patch clamp recordings from the granular cell soma have demonstrated large (5-25 $\mathrm{mV}$ ), all-or-none excitatory electrical postsynaptic potentials (EPSPs) in response to afferent fiber stimulation (Figure 1A). These electrical EPSPs are consistent with the gap junctions observed morphologically (Bell, 1989).

The experiments showed that the amplitude and even the occurrence of the afferent evoked EPSP depends on the granular cell's own membrane potential. The electrical EPSP is absent at depolarizations above $-40 \mathrm{mV}$. As the membrane is hyperpolarized bellow this level, the EPSP grows in a roughly linear manner, but then disappears abruptly with hyperpolarization beyond -90 
$\mathrm{mV}$. In previous experiments, [] antidromic spikes were recorded in the afferent axon, suggesting that the conductance of the gap junction is significant in both directions.

Our hypothesis is that electrical coupling allows the membrane potential of the postsynaptic granular cell to modify the membrane potential of the presynaptic terminal. Under this hypothesis, depolarizing the postsynaptic cell also depolarizes the presynaptic terminal and therefore inactivates sodium channels reducing synaptic current, whereas hyperpolarization beyond $-90 \mathrm{mV}$ blocks the invasion of the spike into the terminal and blocks the synaptic current. The combined effects of the granular cell membrane potential (driving force) and the level of $N a$ channels activation (available conductance) result in the nearly linear relationship between the EPSP size and the holding potential in the range -90 to $-40 \mathrm{mV}$.

We have tested these hypotheses in a compartmental model of the afferent-granular cell circuit built using the NEURON simulation environment (Hines \& Carnevale, 1997).

\section{The model parameters}

Three features of the physiological recordings have guided our construction of the model. First, the spikes recorded at the soma are small $(10-20 \mathrm{mV})$ and the membrane potential is not reset to the resting potential following a spike This implied to us that the spike is generated at a significant electrotonic distance from the soma thus reducing its recorded amplitude and preventing the reset of the soma membrane potential. Second, the cells show outward rectification due presumably to non-inactivating, depolarization activated outward current. Third, the cells have a long time constant (>40 ms) and high input resistance ( $\sim$ 2gigohms) (Zhang et al. , 2006).

The electrotonic distance between the spike generation and the soma suggested a high resistance between the soma and the spike generator in the axon. Accordingly, we inserted an initial segment between the soma and axon with a high resistance and with exclusively passive properties. The axon was assigned active membrane conductances to generate spikes, a fast sodium current $I_{N a}$ and a delayed-rectifier current, $I_{K}$, with maximum conductances set to yield fast repetitive spiking at a rate proportional to the membrane depolarization.

The design of the currents in the soma relied on the second and third features of the physiological data described above. We assigned a non-inactivating potassium current, $I_{K V}$ to the soma and tuned the activation rates and maximum conductance to fit the data. This type of current has been previously identified in cerebellar granule cells (D'Angelo et al. , 2001). The input resistance was measured directly and the capacitance could be determined from the resistance and the time constant. The size of the spikes recorded at the soma was dependent on the initial segment resistance.

The parameters of the potassium current $I_{K V}$ are important for the time course of the membrane voltage at the soma in response to injected current. Consistent with the Hodgkin and Huxley formalism (Hodgkin \& Huxley, 1952), we have described the $I_{K V}$ current as:

$$
I_{K V}=\bar{g}_{K V} n^{4}\left(V-V_{K V r e v}\right)
$$

where activation variable $n$ dependence on membrane voltag $V$ takes the form

$$
\begin{aligned}
\frac{d n}{d t} & =\alpha(V)(1-n)+\beta(V) n \\
\alpha(V) & =\frac{0.13(V+25)}{1-e^{-\frac{V+25}{10}}}, \beta(V)=1.69 e^{-0.0125(V+35)}
\end{aligned}
$$


A

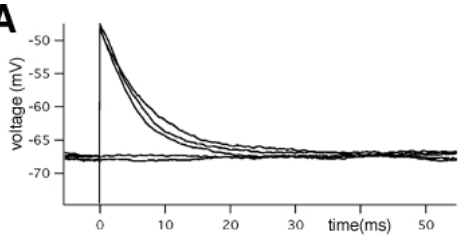

B

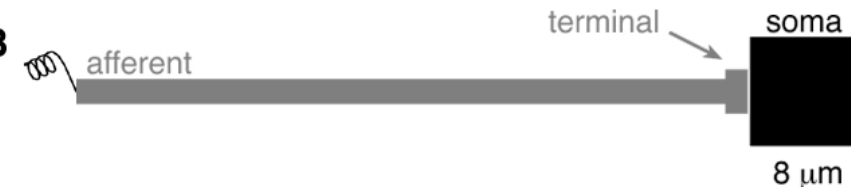

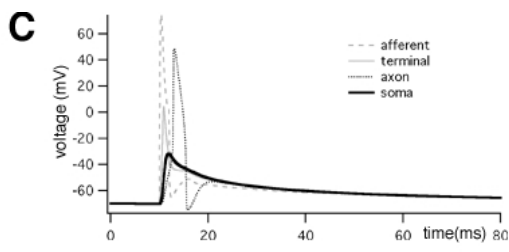

8 um

Figure 1: A. Time course of the electrical EPSP. B. Model morphology. C. Simulated responses to current injection in afferent.

Following (D'Angelo et al. , 2001) we set the reversal potential $V_{K V r e v}=-70 \mathrm{mV}$ and the maximum conductance $\bar{g}_{K V}=0.003$.

To simulate the electrical EPSP, we constructed an afferent with a terminal on the granular cell soma, estimating terminal size from morphological findings (Meek et al., 2001). Hodgkin-Huxley currents were assigned to the model afferent and terminal $\left(I_{N a}, I_{K}\right)$ with maximum conductances to generate a spike when a small current was injected at the distal end of the afferent. The terminal was coupled to the soma with an electrical synapse. The conductance at the synapse was chosen to be large enough to deliver a current injection to the soma of about $1 \mathrm{nA}$ during an afferent spike. Physiological recordings under voltage clamp showed that currents of this magnitude underlie the electrical EPSP.

\section{Results}

We have tuned the model parameters to reflect the electrical properties of the neuronal circuit. All our original choices for parameter values were estimated from cerebellar granle cells (D'Angelo et al. , 2001), which formed the original starting point for our model parameters due to their morphological similarity to the granular cells in the $E L L$. When available, we incorporated data from actual granular cells (Zhang et al. , 2006). As shown in Figure 1, our model cell displays a long decay time constant as seen in the actual granular cell recordings. Based on the experimental results, we have also tuned our model parameters to reflect the current-voltage relationship at the granular soma. The resulting $I-V$ curve is shown in Figure $2 \mathrm{~A}$.

The model simulates many of the properties of the electrical EPSP and its modulation by the membrane potential of the granular cell with only minimal assumptions. An example of a simulated EPSP is shown in figure 1C. Experiments revealed a sharp rise time, large current, and long time constant of the EPSP. Our modelling efforts investigated whether the measured parameters of the cells could give rise to the relationship between the soma membrane voltage at the time of the arrival of afferent spikes and the size of the EPSP.

Our model reproduced the dependence of the EPSP size on the holding membrane voltage, including its abrupt disappearance at about $-90 . \mathrm{mV}$ due to inhibition of the presynaptic spike and the gradual reduction of its size, due mainly to $\mathrm{Na}$ channels inactivation. In Figure $2 \mathrm{~B}$ we have illustrated the relative contribution of the $N a$ channel inactivation vs. simple ohmic dependence on the holding potential. For different holding potentials, we injected a constant amount of current, 

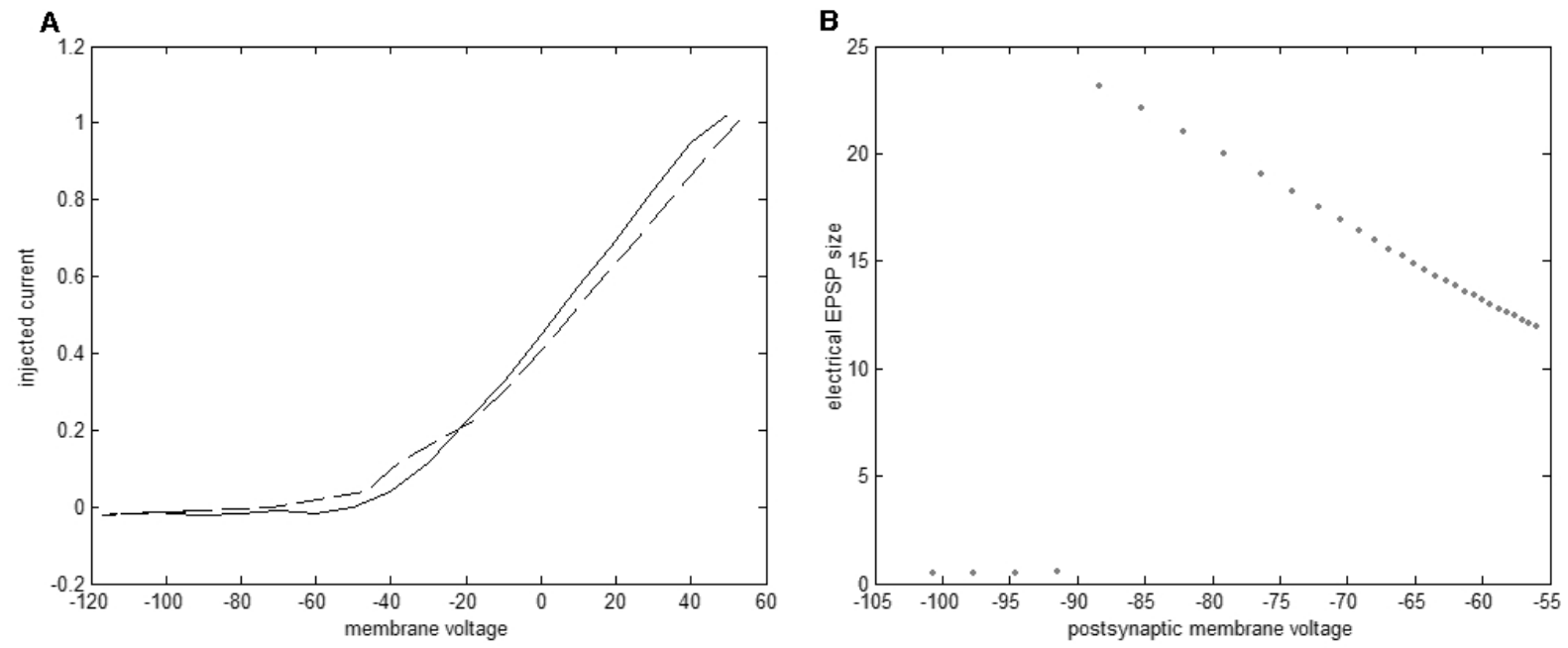

Figure 2: A. Current-voltage relationship in the granular cell model (solid) and experimental data (dashed). B. Model: size of electrical EPSP as a function of postsynaptic membrane voltage. Solid: active $N a$ conductance in the afferent terminal. Dashed: constant current injected in the terminal in lieu of afferent spike.

approximately equivalent with the $N a$ current due to an afferent spike at a holding potential of -90 $\mathrm{mV}$, into the terminal. The resulting EPSP relation to holding potential is due solely to ohmic factors.

\section{Discussion}

Our model replicates the essential features of the experimental data. In particular, we have shown that a reasonable choice of parameters can replicate the dependence of the electrical EPSP size on the postsynaptic potential, including the abrupt disappearance of the EPSP when the granular cell is hyperpolarized. The model thus supports our hypotheses regarding the electrical EPSP and its modulation. The nonlinear interactions shown in the physiological recordings and in the model can result in high temporal sensitivity to the relative timing of the afferent and corollary discharge EPSPs. This sensitivity is required for the precise decoding of afferent latency that is illustrated in behavioral experiments.

The number of spikes generated in the axon of the granular cell depended on several factors: the size of the synaptic current, the duration of the electrical EPSP, the density of sodium channels in the axon and the resistance of the initial segment. Preliminary experimental data suggests that the axonal initiated spiking terminates before the size of the EPSP decays significantly. This suggests that there exists a spike termination mechanism, which we hypothesize is a slow activating, non-inactivating potassium current $I_{K M}$. This current has been shown to be present in cerebellar granule cells (D'Angelo et al. , 2001), where it contributes to the intrinsic bursting and resonance. In the granular cells, this current could contribute to the termination of the burst of spikes, thus playing a central role in the encoding of afferent latency into a spike burst.

In vivo recordings obtained from afferent axons showed antidromic spikes resulting from stim- 
ulation of neighboring afferent receptors []. This raises the possibility that granular cells receive, at each $E O D$, several spikes from a group of neighboring afferents. From our in vitro recordings of granular cells, at least one piece of evidence suggests that the granular cells are capable of functioning in such a regime: distinct granular EPSP's followed afferent stimulation for frequencies up to $500 \mathrm{~Hz}$. Each granular cell could respond to each afferent spike it receives with an EPSP and possibly with an granular spike, since in vitro granular spiking can also have relatively high frequencies (Zhang et al. , 2006). In this context, the dependence relation between the electrical $E P S P$ and the holding potential could translate in fine temporal differences between the latencies of the granular spikes.

Based on morphological observations [], we estimate that each afferent contacts up to 100 granular cells. The connectivity pattern between the afferents and the granular cells, together with our observations about the mechanisms of generation of electrical EPSP's, raise interesting questions about the ways of preserving the temporal sensitivity of the afferents in the context of population coding at the level of granular cells. We will use future in vivo recordings from granular cells to further our understanding of temporal coding in this system.

\section{Acknowledgments}

This material is based upon work supported by the National Science Foundation under Grant No. IOB-0445648.

\section{References}

Bell, Curtis C. 1989. Sensory coding and corollary discharge effects in mormyrid electric fish. J. Exp. Biol., 146, 229-253.

Bell, Curtis C., Grant, Kirsty, \& Serrier, Jacques. 1992. Sensory processing and corollary discharge effects in the mormyromast regions of the mormyrid electrosensory lobe: I. Field potentials and cellular activity in associated structures. J. Neurophysiol., 68, 843-858.

D’Angelo, E., Nieus, T., Maffei, A., Armano, S., Rossi, P., Taglietti, V., Fontana, A., \& Naldi, G. 2001. Theta-frequency bursting and resonance in cerebellar granule cells: Experimental evidence and modeling of a slow K+-dependent mechanism. J. Neurosci., 21, 759-770.

Hall, C., Bell, C. C., \& Zelick, R. 1995. Behavioral evidence of a latency code for stimulus intensity in mormyrid electric fish. J. Comp. Physiol. A, 177, 29-39.

Hines, M., \& Carnevale, T. 1997. The NEURON simulation environment. Neural Comput., 9, 1179-1209.

Hodgkin, A. L., \& Huxley, A. F. 1952. A quantitative description of membrane current and its application to conduction and excitation in nerve. J. Physiol. (London), 108, 37-77.

Meek, Johannes, Hafmans, Theo G. M., Hans, Victor, Bell, Curtis C., \& Grant, Kirsty. 2001. Myelinated dendrites in the mormyrid electrosensory lobe. J. Comp. Neurol., 431, 255-275.

Zhang, Jianmei, Han, Victor Z., Meek, Johannes, \& Bell, Curtis C. 2006. Granular Cells of the Mormyromid Electrosensory Lobe and Postsynaptic Controlover the Efficacy of and Electrical Synapse. unpublished. 